\title{
Expression of signal transducer and activator of transcription 3 and its phosphorylated form is significantly upregulated in patients with papillary thyroid cancer
}

\author{
LI YAN $^{1}$, LI LI $^{2}$, QINGHUAI LI ${ }^{1}$, WANG DI ${ }^{1}$, WEI SHEN ${ }^{1}$, LINLEI ZHANG ${ }^{1}$ and HAO GUO ${ }^{1}$ \\ ${ }^{1}$ Department of Thyroid and Breast Surgery, The Second Hospital Affiliated to Hebei Medical University, Shijiazhuang, \\ Hebei 050000; ${ }^{2}$ Department of Histology and Embryology, Hebei Medical University, \\ Shijiazhuang, Hebei 050071, P.R. China
}

Received June 12, 2014; Accepted April 2, 2015

DOI: $10.3892 /$ etm.2015.2409

\begin{abstract}
The aim of the present study was to investigate the expression of signal transducer and activator of transcription 3 (STAT3) and phosphorylated STAT3 (pSTAT3) in tissues of papillary thyroid cancer (PTC) in comparison with the expression in adjacent normal tissues. The expression of STAT3, pSTAT3, fibroblast growth factor 2 (FGF2) and vascular endothelial growth factor-C (VEGF-C) was examined in tissues of 42 cases of PTC and the adjacent normal tissues of 20 of the 42 PTC cases using immunohistochemistry and western blotting. The association between the expression levels and the clinicopathological features was analyzed. The expression of STAT3, pSTAT3, FGF2 and VEGF-C in the PTC tissues (76.2, $42.9,81.0$ and $73.8 \%$, respectively) was significantly higher than that in the normal tissues $(\mathrm{P}<0.05)$. In the PTC tissues, the expression of STAT3 was linearly correlated with the levels of pSTAT3 and VEGF-C $(\mathrm{P}<0.05)$. In conclusion, STAT3 and pSTAT3 are significantly upregulated in PTC tissues, and may potentially be used as markers to screen for PTC with lymph node metastasis.
\end{abstract}

\section{Introduction}

Thyroid carcinoma is the most common malignant tumor in the human neck, and its incidence is increasing markedly worldwide (1). Papillary thyroid cancer (PTC) is the most frequently observed thyroid carcinoma, accounting for $>90 \%$ of all thyroid carcinoma cases, and cervical lymph node metastasis is very common in patients with PTC $(2,3)$. Cervical lymph

Correspondence to: Dr Li Yan, Department of Thyroid and Breast Surgery, The Second Hospital Affiliated to Hebei Medical University, 215 Heping West Road, Shijiazhuang, Hebei 050000, P.R. China

E-mail: gxfcyl@163.com

Key words: STAT3, pSTAT3, FGF2, VEGF-C, papillary thyroid cancer node metastasis is an important risk factor for PTC recurrence rate and survival rate reduction. The early detection of cervical lymph node metastasis is therefore the key to improving the cure rate and to reducing the recurrence of PTC $(4,5)$. Currently, ultrasound-guided fine-needle biopsy (US-guided FNAB) is the most accurate and cost-effective method for evaluating cervical lymph node metastasis (6). However, 6-8\% of results obtained by US-guided FNAB are false negatives (7). In addition, US-guided FNAB is operator-dependent (8). Numerous recent studies have demonstrated that fine-needle aspirate washout thyroglobulin (FNA wash-out $\mathrm{Tg}$ ) can also be used to identify metastatic lymph nodes $(9,10)$; however, the diagnostic threshold of FNA wash-out Tg has yet to be established (10). Currently, the method of detecting specific tumor molecular markers is having an increasing effect on the diagnosis of malignancies.

Signal transducer and activator of transcription 3 (STAT3) is a cancer gene. STAT3 and its phosphorylated form, pSTAT3, have pivotal roles in the processes of invasion and metastasis in malignant tumors (11). Fibroblast growth factor 2 (FGF2) is a growth factor that can promote angiogenesis (12). FGF2 is important in tumor lymphangiogenesis and lymph node metastasis. Vascular endothelial growth factor-C (VEGF-C) is involved in angiogenesis of a variety of malignant tumors (13). Following activation, STAT3 induces the expression of VEGF, thus promoting tumor cell proliferation and angiogenesis.

The aim of the present study was to examine the expression of STAT3, pSTAT3, FGF2 and VEGF-C in the cancerous and adjacent normal thyroid tissues of patients with PTC using immunohistochemistry and western blotting, and to explore the association among STAT3, pSTAT3, FGF2 and VEGF-C.

\section{Materials and methods}

Patients. A total of 42 patients with PTC who were admitted to the Second Hospital Affiliated to Hebei Medical University (Shijiazhuang, China) between January 2012 and August 2013 were enrolled in the present study. The patients included 33 females and nine males, with an average age of 43.5 years (range, 20-65 years). The diseases were pathologically confirmed following surgery. The normal thyroid glands 
$\geq 2 \mathrm{~cm}$ away from the edge of the tumor in 20 patients, who were randomly selected from the 42 cases, were used as controls. None of the patients had received anti-tumor therapy before the surgery. Prior written and informed consent was obtained from every patient and the study was approved by the Ethics Review Board of the Second Hospital Affiliated to Hebei Medical University.

Immunohistochemical staining. Immunohistochemistry was performed using a general rat/rabbit immunohistochemical kit (Shanghai Gene Co., Ltd., Shanghai, China) using a two-step procedure. The specimens were fixed with $4 \%$ paraformaldehyde for $48 \mathrm{~h}$ and embedded in paraffin. The paraffin-embedded tissue was sliced continuously into 3-5- $\mu \mathrm{m}$ sections, and the sections were deparaffinized and rehydrated. Antigen retrieval was conductedby incubating with sodium citrate for $20 \mathrm{~min}$ and endogenous peroxidase activity was blocked with $3 \%$ hydrogen peroxide in methanol for $5 \mathrm{~min}$ at room temperature. Non-specific binding was blocked by incubating the slides in $10 \%$ goat serum for $15 \mathrm{~min}$ at room temperature. The sections were then incubated overnight at $4^{\circ} \mathrm{C}$ with primary antibody. The primary antibodies and dilution rates were as follows: Mouse anti-human monoclonal STAT3 (1:200; cat. no. AO1236a; Abgent, San Diego, CA, USA), rabbit anti-human polyclonal pSTAT3 (1:200; cat. no. AP3261a; Abgent), rabbit anti-human polyclonal FGF2 (1:50; cat. no. 11234-1-AP; Proteintech, San Diego, CA, USA) and rabbit anti-human monclonal VEGF-C (1:10; cat. no. AJ1813a Abgent). Following incubation, the sections were placed at room temperature for $30 \mathrm{~min}$ and incubated with biotinylated anti-mouse (1:100; Abcam, Cambridge, UK) and anti-rabbit (1:100; Abcam, Cambridge, UK) secondary antibodies at room temperature for $45 \mathrm{~min}$. The slides were developed using a 3,3'-diaminobenzidine kit (Shanghai Gaochuang Chemical Technology Co., Ltd., Shanghai, China), counterstained with hematoxylin, dehydrated through a graded alcohol series and permeabilized in xylene. Images of the slides were captured using a light microscope. The primary antibody was replaced by phosphate-buffered saline in the blank controls. Image analysis was carried out using Image-Pro software (Media Cybernetics, Inc., Rockville, MD, USA). Measurements were performed in triplicate for three separate sections of the tumor and averaged to minimize heterogeneity within the tumors. Negative results were defined as an absence of staining in the cytoplasm and staining of $<10 \%$ of the nuclei. A three-point scale, based on intensity, was utilized in the scoring of cytoplasmic staining, as follows: 0 , negative; $1+$, weakly positive; $2+$, strongly positive. When staining was present but not found in the cytoplasm, nuclear staining was judged as positive when $>10 \%$ of the nuclei showed positive staining. For pSTAT3, the presence of any areas with $>10 \%$ tumor cells showing definitive nuclear staining resulted in a positive score.

Western blot analyses. Tissue (100 mg) from each sample was collected and homogenized with $1 \mathrm{ml}$ lysis buffer. The specimens were then centrifuged at 22,580 x g for $15 \mathrm{~min}$ at $4^{\circ} \mathrm{C}$ for the preparation of the total protein. Proteins (100 $\mu \mathrm{g} / \mathrm{lane})$ were separated using sodium dodecyl sulfate-polyacrylamide gel electrophoresis on precast $10 \%$ polyacrylamide gels and transferred electrophoretically to polyvinylidene difluoride
Table I. Expression of STAT3, pSTAT3, FGF2 and VEGF-C in the PTC and adjacent normal tissues, as determined by immunohistochemical staining.

\begin{tabular}{lccc}
\hline & & \multicolumn{2}{c}{ Positive (n) } \\
\cline { 3 - 4 } Tissue type & Negative (n) & $1+$ & $2+$ \\
\hline Adjacent normal & & & \\
STAT3 & 12 & 8 & 0 \\
pSTAT3 & 20 & 0 & 0 \\
FGF2 & 11 & 9 & 0 \\
VEGF-C & 13 & 7 & 0 \\
PTC & & & \\
STAT3 & 10 & 12 & 20 \\
pSTAT3 & 24 & 7 & 11 \\
FGF2 & 8 & 19 & 15 \\
VEGF-C & 11 & 13 & 18 \\
\hline
\end{tabular}

Adjacent normal, n=20; PTC, $\mathrm{n}=42.1+$, weakly positive; $2+$, strongly positive; PTC, papillary thyroid cancer; STAT3, signal transducer and activator of transcription 3; pSTAT3, phosphorylated STAT3; FGF2, fibroblast growth factor 2; VEGF-C, vascular endothelial growth factor-C.

membranes. The membranes were blocked in 5\% bovine serum albumin for $1 \mathrm{~h}$ at room temperature and then probed overnight at $4^{\circ} \mathrm{C}$ with the appropriate primary antibodies, as follows: Mouse anti-human monoclonal STAT3 (1:500;cat. no. AO1236a; Abgent), rabbit anti-human polyclonal pSTAT3 (1:500; cat. no. AP3261a; Abgent), rabbit anti-human polyclonal FGF2 (1:1,000; cat. no. 11234-1-AP; Proteintech), rabbit anti-human monoclonal VEGF-C (1:100; cat. no. AJ1813a Abgent) or rabbit anti-human polyclonal $\beta$-actin $(1: 2,000$; cat. no. ab8227; Abcam). The membranes were subsequently incubated with secondary goat anti-rabbit immunoglobulin $\mathrm{G}$ $(1: 5,000$; Proteintech) at room temperature for $2 \mathrm{~h}$. Target proteins were visualized using the enhanced chemiluminescence detection system (Millipore, Billerica, MA, USA). Images were obtained using a transmission scanner, with $\beta$-actin as a reference protein. The image analysis was performed using ImageJ software (US National Institutes of Health, Bethesda, MD, USA).

Statistical analysis. Statistical analyses were performed using SPSS version 19.0 (IBM-SPSS, Armonk, NY, USA). $\chi^{2}$ tests were performed to determine the statistical significance of any association between the variables. Statistical significance was defined as a P-value of $<0.05$.

\section{Results}

Differential expression of STAT3 and pSTAT3 in the PTC and adjacent normal tissues. To determine the expression of STAT3 and pSTAT3 in the PTC tissues of the 42 patients, immunohistochemical staining was performed, as shown in Fig. 1. The adjacent normal tissues of 20 out of the 42 patients were also examined to serve as the controls. The positive 

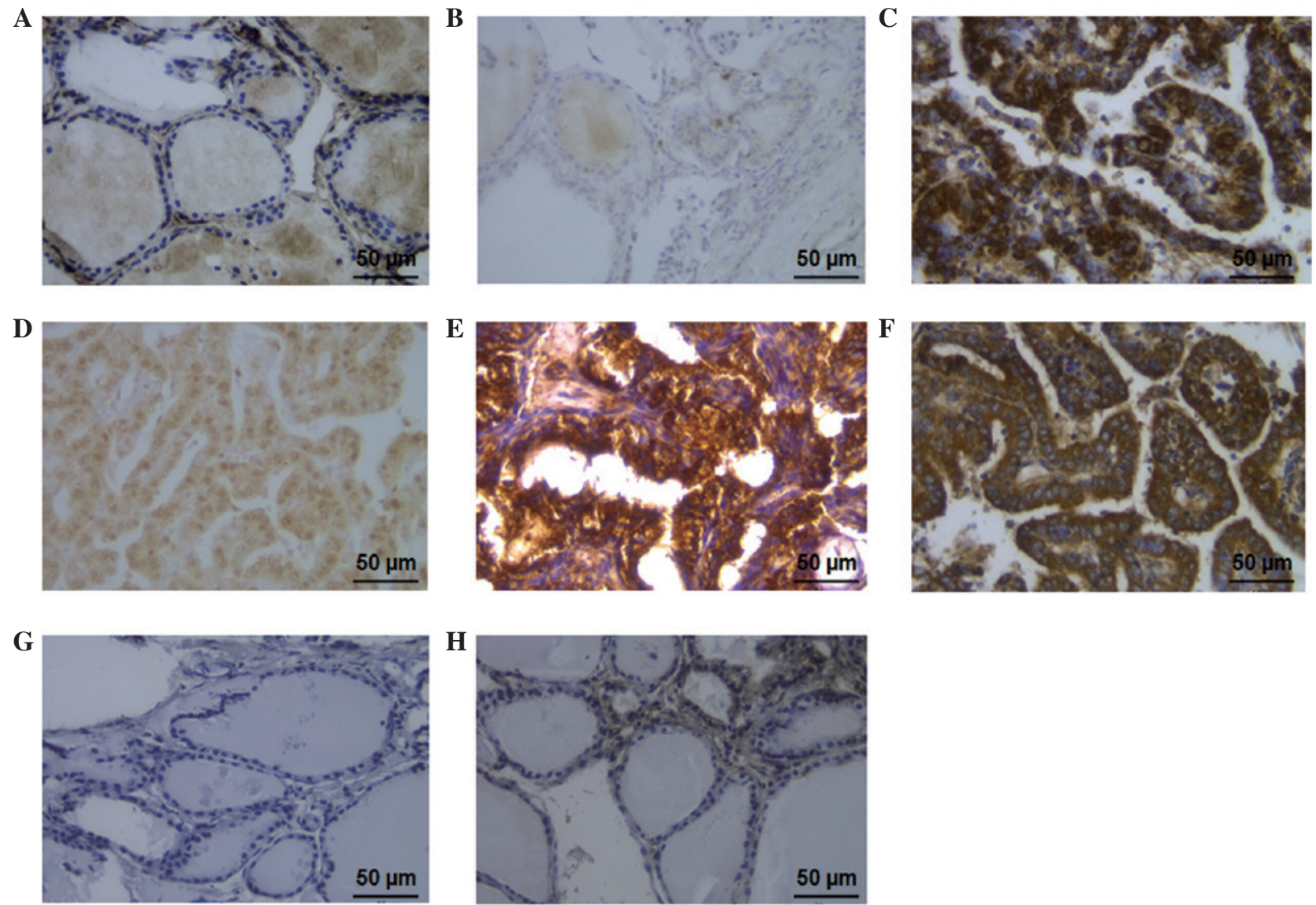

Figure 1. Immunohistochemical staining (magnification, $\mathrm{x} 400$ ). The specimens were fixed with $4 \%$ paraformaldehyde for $48 \mathrm{~h}$ and embedded in paraffin. The paraffin-embedded tissue was sliced continuously into 3-5- $\mu$ m sections. Non-specific binding was blocked by incubating the slides in $10 \%$ goat serum for $15 \mathrm{~min}$ at room temperature. The sections were then incubated overnight at $4^{\circ} \mathrm{C}$ with primary antibody. (A) STAT3 and (B) pSTAT3 expression in adjacent normal tissues; (C) STAT3 and (D) pSTAT3 expression in PTC tissues; (E) FGF2 and (F) VEGF-C expression in PTC tissues; (G) FGF2 and (H) VEGF-C expression in adjacent normal tissues. STAT3, signal transducer and activator of transcription 3; pSTAT3, phosphorylated STAT3; FGF2, fibroblast growth factor 2; VEGF-C, vascular endothelial growth factor-C; PTC, papillary thyroid cancer.

immunocytochemical signal pattern for the STAT3 protein was indicated by the presence of brownish yellow granules in the cytoplasm (Fig. 1A and C). pSTAT3 expression, which was indicated by brownish yellow staining, was mainly located in the nucleus (Fig. 1B and D). The expression rates of STAT3 and pSTAT3 protein in the PTC tissues were 76.2 and $42.9 \%$, respectively; however, in the normal thyroid tissues the expression rates were 40.0 and $0.0 \%$, respectively (Table I and Fig. 1). These results suggested that the expression rates of STAT3 and pSTAT3 protein in the PTC tissues were significantly different from those in the normal control tissues.

FGF2 is an upstream cytokine that regulates the STAT3 signal transduction pathway. VEGF-C serves as a downstream protein regulator of STAT3 and promotes lymphangiogenesis by activating the VEGF receptor 3 (VEGFR-3). To confirm the above results, the expression of FGF2 and VEGF-C in the PTC and adjacent tissues was detected (Fig. 1). The expression rate of FGF2 in the PTC tissues was $81.0 \%$, compared with $45.0 \%$ in the adjacent normal tissues, suggesting a significantly higher rate in the PTC tissues than that in the normal group $(\mathrm{P}<0.05)$ (Table I and Fig. $1 \mathrm{E}$ and $\mathrm{G})$. The positive immunocytochemical signal pattern for the VEGF-C protein was the presence of brownish yellow granules in the cytoplasm. The expression rate in the PTC group was $73.8 \%$ (Fig. 1F), compared with $35.0 \%$ in the adjacent normal tissues
(Fig. 1H); therefore, the VEGF-C protein levels in the PTC tissues were significantly higher than those in the normal tissues $(\mathrm{P}<0.05)$. In summary, the expression levels of STAT3 and pSTAT3 were significantly increased in the PTC tissues when compared with those in the adjacent normal tissues.

As shown in Table II, STAT3, pSTAT3, FGF2 and VEGF-C expression was not associated with the patient age, gender, tumor size or number of lesions ( $\mathrm{P}>0.05)$; however, notable correlation was found between the expression of these proteins and cancer development, such as lymph node metastasis $(\mathrm{P}<0.05)$, which is consistent with the results in Fig. 1.

Western blot analyses of STAT3 and pSTAT3 expression in the PTC and adjacent normal tissues. To investigate whether the STAT3 and pSTAT3 proteins exhibited differential expression in the PTC and adjacent normal tissues, the total proteins were extracted and subjected to western blot analysis, with the cellular $\beta$-actin protein serving as a loading control. Since FGF2 is an upstream cytokine regulating the STAT3 signal transduction pathway and VEGF-C is a downstream protein for the regulation of STAT3, the expression of these proteins was also detected using western blot analyses in this study. Representative blots are shown in Fig. 2A. The mean normalized optical density (OD) of the protein bands 


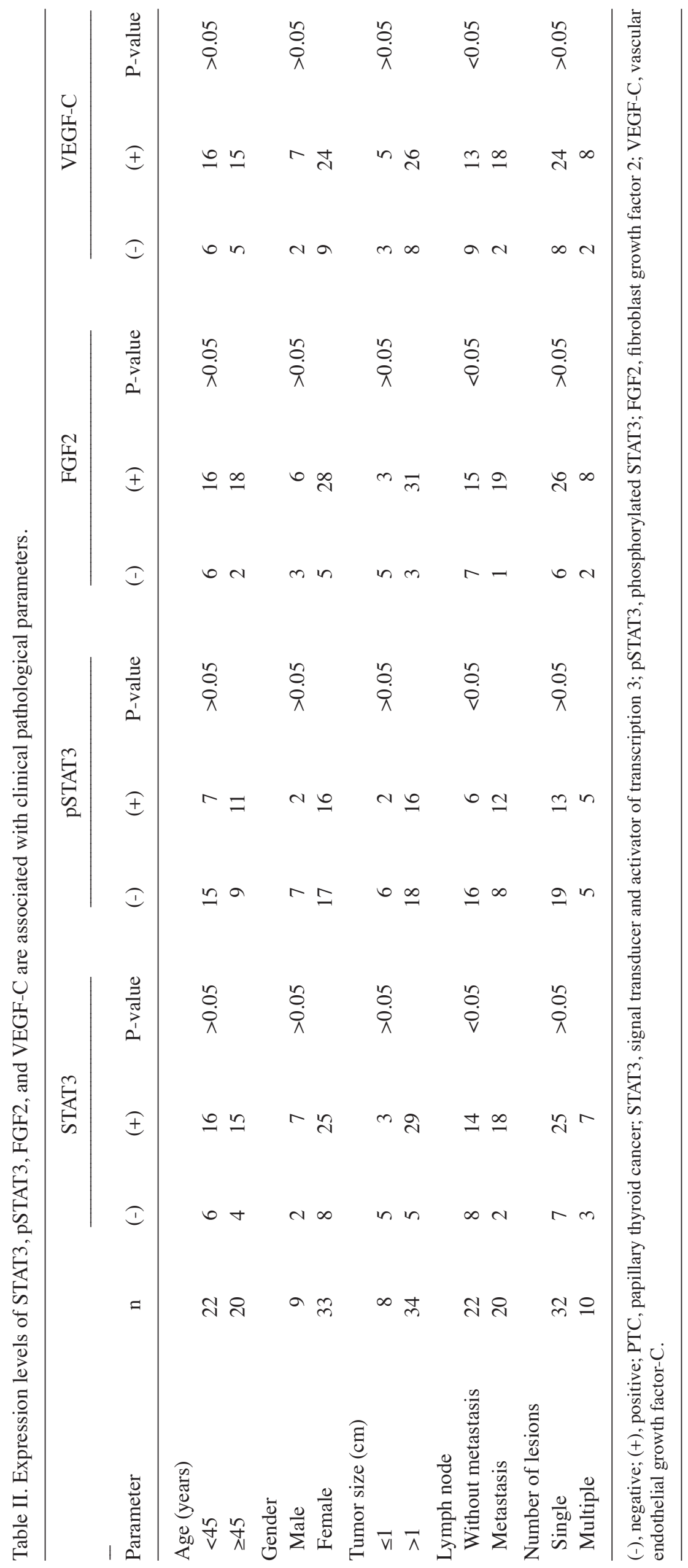


A

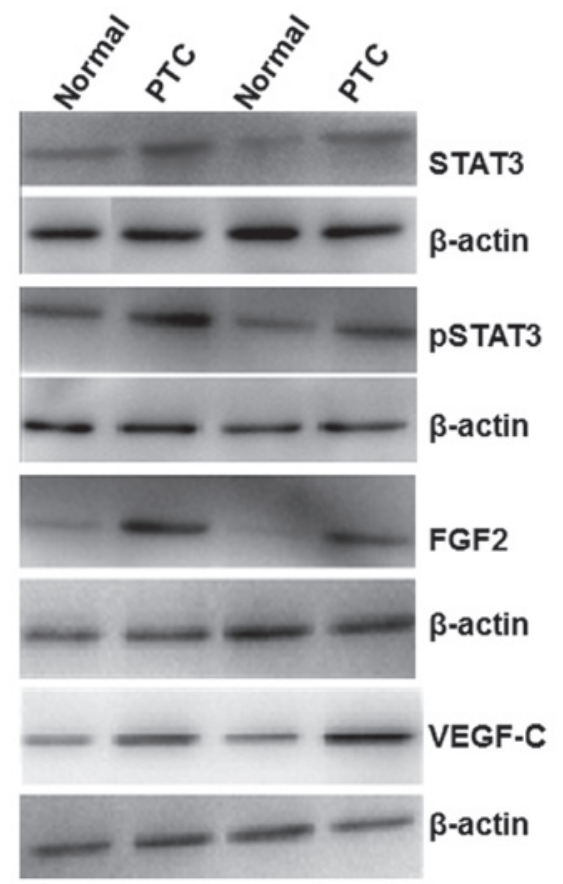

B

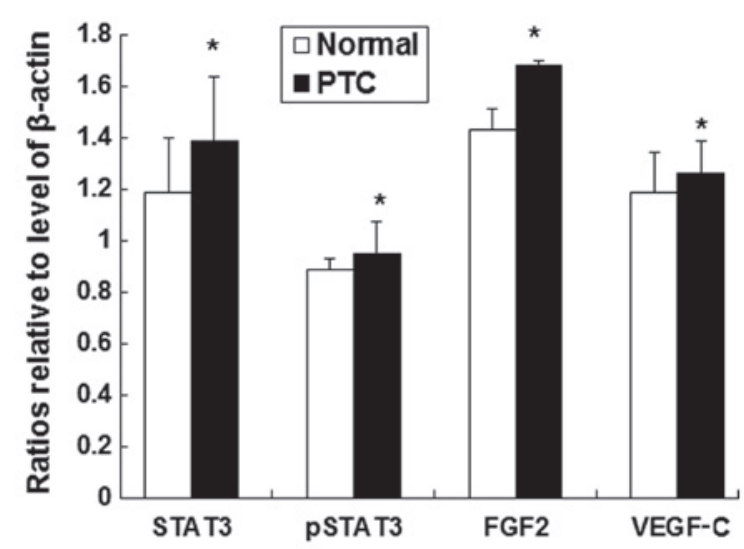

Figure 2. Expression of STAT3, pSTAT3, FGF2 and VEGF-C proteins as determined by western blotting. (A) Tissue (100 mg) from each sample was collected and homogenized with $1 \mathrm{ml}$ lysis buffer. Proteins (100 $\mu \mathrm{g} / \mathrm{lane})$ were separated using sodium dodecyl sulfate-polyacrylamide gel electrophoresis on precast $10 \%$ polyacrylamide gels and transferred electrophoretically to polyvinylidene difluoride membranes. The following primary antibodies were used: Rabbit anti-human STAT3 (1:500), rabbit anti-human pSTAT3 (1:500), rabbit anti-human FGF2 (1:1,000), rabbit anti-human VEGF-C (1:100) or rabbit anti-human $\beta$-actin. The membranes were then incubated with secondary goat anti-rabbit antibody $(1: 5,000)$ at room temperature for 2 h. Target proteins were visualized using the enhanced chemiluminescence detection system, and images were obtained using a transmission scanner, with $\beta$-actin as a reference protein. (B) Histograms showing the mean normalized OD of the detected protein bands relative to the OD of the $\beta$-actin band. Error bars show the standard error of the mean. "P<0.05 when compared with the normal controls. STAT3, signal transducer and activator of transcription 3; pSTAT3, phosphorylated STAT3; FGF2, fibroblast growth factor 2; VEGF-C, vascular endothelial growth factor-C; PTC, papillary thyroid cancer; OD, optical density.

relative to the OD of the $\beta$-actin band from each protein was calculated, and statistical analysis was performed (Fig. 2B).

As shown in Fig. 2A and B, the mean levels of STAT3 and pSTAT3 proteins, as well as the levels of FGF2 and VEGF-C, were significantly increased in the PTC tissues in comparison with the levels in the adjacent normal tissues $(\mathrm{P}<0.05)$. These results suggest that the increased levels of STAT3 and pSTAT3 may be associated with the development of PTC.

Through Pearson correlation analysis it was found that the expression of STAT3 and pSTAT3 exhibited a linear correlation in the PTC tissues $(\mathrm{r}=0.662, \mathrm{P}<0.05)$. Furthermore, the expression of STAT3 and VEGF-C showed a linear correlation $(\mathrm{r}=0.733, \mathrm{P}<0.05)$. These results are consistent with the finding that increased levels of STAT3 and pSTAT3 may be associated with the development of PTC.

\section{Discussion}

Cervical lymph node metastasis is an important risk factor that can increase the PTC recurrence rate and reduce the survival rate (3). According to statistics, 20-90\% of patients with PTC have already developed cervical lymph node metastasis before they are diagnosed (14). Cervical lymph node metastasis mostly occurs in the VI region. Between 28 and $33 \%$ of cervical lymph node metastasis cases are not found by preoperative radiological examination and surgical treatment but are confirmed following prophylactic VI cleaning (15). At present, there is no ideal method to determine whether a patient exhibits lymph node metastasis prior to surgery, and potential lymph node metastasis or micrometastasis can be hard to find in preoperative routine examination. An improvement in the preoperative diagnosis rate for cervical lymph node metastasis is therefore of vital significance and could reduce the repeated surgery rate, avoid the patient being subjected to excessive surgery and improve the survival rate. Equally, improved methods of preoperative diagnosis would be applicable to patients who are difficult to diagnose using preoperative fine-needle aspiration biopsy (FNA). At present, the method of detecting specific tumor molecular markers is playing an increasingly important role in the diagnosis of malignant tumors. The ability to determine whether a patient with thyroid carcinoma exhibits cervical lymph node metastasis through the detection of the aforementioned molecular markers is likely to provide an important contribution to the improvement of the surgical treatment of thyroid cancer.

Janus protein tyrosine kinase (JAK)]-STAT pathways are important intracellular signal transduction pathways that are associated with tumor occurrence, development and metastasis $(16,17)$. The JAK-STAT3 pathway can be activated by a variety of extracellular signal proteins, including cytokines, epidermal growth factor and non-receptor tyrosine kinases. JAKs are activated by the binding of the ligand to the receptor. The JAKs then phosphorylate tyrosine residues on the receptor with enhanced kinase activity, and sites are created for interactions with proteins containing phosphotyrosine-binding domains (18). STAT3 proteins, which have 
phosphotyrosine residue-binding domains, are recruited to the receptors where they are also tyrosine-phosphorylated. The phosphorylated STAT3 proteins then translocate into the cell nucleus and bind to target genes (19). This affects a series of associated basic cell functions, including factor activation, protein expression, tumor progression and metastasis.

STAT3 expression increases in a variety of malignant tumor types, such as breast, pancreatic, lung and ovarian cancer and melanoma, and plays a crucial role in tumorigenesis, tumor angiogenesis, invasion and metastasis (20-22). The STAT3 signal transduction pathway may perform a vital role in the tumor microenvironment of immune tolerance. According to early studies, STAT3 can inhibit the differentiation and maturation of dendritic cells, induce T-cell immune tolerance and promote the secretion of certain immunosuppressive cytokines, such as transforming growth factor- $\beta$, interleukin (IL)-10, IL- 6 and VEGF, thereby promoting tumor growth (23-26). Chen et al (27) found that the expression of STAT3 and pSTAT3 in breast cancer tissues was significantly higher than that in paracancerous tissues, and that the expression level of STAT3 was closely associated with axillary lymph node metastasis. It was therefore proposed in the same study that STAT3 could be used as a predictor of breast cancer postoperative survival rate. In a study by Bednarek et al (28), it was found that STAT3 could significantly inhibit the growth of the bladder cancer cell line T24 and induce apoptosis through RNA interference silencing. There are, however, different viewpoints: A previous study (29) showed that the expression of pSTAT3 in tumor cells was inversely correlated with tumor diameter, vascular invasion and distant metastasis. Furthermore, pSTAT3 can inhibit tumor growth, and the pSTAT3 expression level of inpatients with pulmonary metastasis and bone metastasis of thyroid carcinoma has been found to be lower than that of those without metastasis (29).

The results of the present study showed that STAT3 was expressed in the cytoplasm of the PTC and adjacent normal tissues. The expression of pSTAT3 was solely found in the nuclei of the PTC tissues. The expression of STAT3 and pSTAT3 in the PTC tissues was significantly higher than that in the paracancerous tissues, and the expression levels in the PTC with lymph node metastasis group were significantly higher than those in the PTC without lymph node metastasis group. The trends of the two were accordant. This showed that STAT3 and pSTAT3 play important roles in promoting the occurrence, development and lymph node metastasis of PTC. The expression levels were not found to be associated with age, gender, tumor size or the number of lesions.

PTC tumor cells induce local blood vessel and lymphatic regeneration, and then promote cervical lymph node metastasis. FGF2, a type of multipotent cell growth factor, is an important upstream cytokine regulator of the STAT3 signal transduction pathway that is widely distributed in a variety of tissues and organs derived from the mesoderm and neuroectoderm, as well as in tumor tissue. FGF2 activates target cell FGFRs predominantly by autocrine or paracrine means, causes a series of intracellular signal transduction pathways and plays an important role in promoting tumor angiogenesis, lymphangiogenesis and lymph node metastasis (30-33).
VEGF-C is the only currently known lymph-specific angiogenic factor. VEGF-C can combine with VEGFR-3 and induce the phosphorylation of VEGFR-3, which causes lymphatic endothelial cell proliferation. VEGF-C, as a downstream protein of regulator STAT3, plays an important role in tumor angiogenesis. The VEGF-C expression level has been shown to be positively associated with blood vessel density and lymph node metastasis in tumor tissues $(13,34,35)$. In a study by Karaca et al (36) it was reported that the expression levels of serum VEGF in patients with thyroid carcinoma were significantly increased; VEGF can therefore be used as a predictor of cervical lymph node metastasis and local recurrence of PTC. The preoperative serum VEGF-C level can be used as a preoperative diagnostic predictor irrespective of whether a patient exhibits cervical lymph node metastasis (37). Furthermore, the level of VEGF-C is closely associated with tumor size, lymph node metastasis and distant metastasis.

The results of the present study showed that the expression of FGF2 and VEGF-C in the PTC group was higher than that in the adjacent normal tissues. Additionally, the expression levels in the PTC with lymph node metastasis group were higher than those in the PTC without lymph node metastasis group. This observation is consistent with the results of former studies $(37,38)$, indicating that FGF2 and VEGF-C may promote local tissue angiogenesis and lymphangiogenesis in PTC, thus promoting PTC formation, local infiltration and lymph node metastasis; however, there is no correlation in patients with PTC between FGF2 and VEGF-C expression and age, gender, tumor size or the number of lesions.

The results of the present correlation analysis showed that STAT3 and pSTAT3 expression was higher in PTC tissues and was positively correlated with FGF2 and VEGF-C expression. This indicates that STAT3 and pSTAT3 enhance lymphatic and vascular density and play a significant role in the local invasion and lymph node metastasis associated with PTC. The detection of STAT3 and pSTAT3 is simple and applicable to patients who cannot be diagnosed using preoperative FNA. In conclusion, STAT3 and pSTAT3, two novel immunohistochemical markers, may represent a valid adjunctive tool for screening PTC with lymph node metastasis.

\section{Acknowledgements}

This study was supported by the Hebei Province Science and Technology Support Program (grant no. 10246140D) of China.

\section{References}

1. Kent WD, Hall SF, Isolato PA, Houlden RL, George RL and Groome PA: Increased incidence of differentiated thyroid carcinoma and detection of subclinical disease. CMAJ 177: 1357-1361, 2007.

2. Alvarado R, Sywak MS, Delbridge L and Sidu SB: Central lymph node dissection as a secondary procedure for papillary thyroid cancer: Is there added morbidity? Surgery 145: 514-518, 2009.

3. Lee YS, Lim YS, Lee JC, Wang SG, Kim IJ and Lee BJ: Clinical implication of the number of central lymph node metastasis in papillary thyroid carcinoma: preliminary report. World $\mathbf{J}$ Surg 34: 2558-2563, 2010

4. Wan H, Zhang B, Liu S, et al: Preliminary study of patterns of level IIb lymph node metastasis in papillary thyroid carcinoma. Zhonghua Er Bai Yan Hou Tou Jing Wai Ke Za Zhi 49: 27-30, 2014 (In Chinese). 
5. Eun YG, Lee YC and Kwon KH: Predictive factors of contralateral paratracheal lymph node metastasis in papillary thyroid cancer: prospective multicenter study. Otolaryngol Head Neck Surg 150: 210-215, 2014.

6. Cooper DS, Doherty GM, Haugen BR, et al; American Thyroid Association Guidelines Taskforce: Management guidelines for patients with thyroid nodules and differentiated thyroid cancer. Thyroid 16: 109-142, 2006.

7. Frasoldati A, Toschi E, Zini M, et al: Role of thyroglobulin measurement in fine-needle aspiration biopsies of cervical lymph nodes in patients with differentiated thyroid cancer. Thyroid 9: 105-111, 1999.

8. Choi JS, Kim J, Kwak JY, Kim MJ, Chang HS and Kim EK: Preoperative staging of papillary thyroid carcinoma: comparison of ultrasound imaging and CT. AJR Am J Roentgenol 193: 871-878, 2009.

9. Kim MJ, Kim EK, Kim BM, et al: Thyroglobulin measurement in fine-needle aspirate washouts: the criteria for neck node dissection for patients with thyroid cancer. Clin Endocrinol (Oxf) 70:145-151, 2009.

10. Pacini F, Fugazzola L, Lippi F, et al: Detection of thyroglobulin in fine needle aspirates of nonthyroidal neck masses: a clue to the diagnosis of metastatic differentiated thyroid cancer. J Clin Endocrinol Metab 74: 1401-1404, 1992.

11. ZhangZ,Bai Y,LiP,ZhaoJ,Wang Y,Sun Land Tang J:Relationship between activated STAT3 protein and epithelial-mesenchymal transition in papillary thyroid carcinonma. Lin Chung Er Bi Yan Hou Tou Jing Wai Ke Za Zhi 27: 1265-1268, 2013 (In Chinese).

12. Slattery ML, John EM, Stern MC, et al: Associations with growth factor genes (FGF1, FGF2, PDGFB, FGFR2, NRG2, EGF, ERBB2) with breast cancer risk and survival: the Breast Cancer Health Disparities Study. Breast Cancer Res Treat 140: 587-601, 2013.

13. Jebreel A, England J, Bedford K, Murphy J, Karsai L and Atkin S Vascular endothelial growth factor (VEGF), VEGF receptors expression and microvascular density in benign and malignant thyroid diseases. Int J Exp Pathol 88: 271-277, 2007.

14. Dralle H and Machens A: Surgical approaches in thyroid cancer and lymph-node metastases. Best Pract Res Clin Endocrinol Metab 22: 971-987, 2008

15. Sakorafas GH, Sampanis D and Safioleas M: Cervical lymph node dissection in papillary thyroid cancer: current trends, persisting controversies, and unclarified uncertainties. Surg Oncol 19: e57-e70, 2010.

16. Bournazou E and Bromberg J: Targeting the tumor microenvironment: JAK-STAT3 signaling. JAKSTAT 2: e23828, 2013.

17. Voskas D, Ling LS and Woodgett JR: Signals controlling un-differentiated states in embryonic stem and cancer cells: role of the phosphatidylinositol 3' kinase pathway. J Cell Physiol 229: 1312-1322, 2014

18. Zouein FA, Duhé RJ and Booz GW: JAKs go nuclear: emerging role of nuclear JAK1 and JAK2 in gene expression and cell growth. Growth Factors 29: 245-252, 2011

19. Kamran MZ, Patil P and Gude RP: Role of STAT3 in cancer metastasis and translational advances. Biomed Res Int 2013 421821, 2013

20. Feng Y, Ke C, Tang Q, et al: Metformin promotes autophagy and apoptosis in esophageal squamous cell carcinoma by downregulating Stat3 signaling. Cell Death Dis 5: e1088, 2014.

21. Pandurangan AK and Esa NM: Signal transducer and activator of transcription 3 - a promising target in colitis-associated cancer. Asian Pac J Cancer Prev 15: 551-560, 2014.
22. Zhang J, Gill A, Atmore B, Johns A, Delbridge L, Lai R and McMullen T: Upregulation of the signal transducers and activators of transcription 3 (STAT3) pathway in lymphatic metastases of papillary thyroid cancer. Int J Clin Exp Pathol 4: 356-362, 2011.

23. Kawakami Y, Yaguchi T, Sumimoto H, et al: Improvement of cancer immunotherapy by combing molecular targeted therapy. Front Oncol 3: 136, 2013

24. Gu L, Talati P, Vogiatzi P, et al: Pharmacological suppression of JAK $1 / 2$ by JAK $1 / 2$ inhibitor AZD1480 potently inhibits IL-6-induced experimental prostate cancer metastases formation. Mol Cancer Ther 13: 1246-1258, 2014.

25. Farren MR, Carlson LM, Netherby CS, et al: Tumor-induced STAT3 signaling in myeloid cells impairs dendritic cell generation by decreasing PKC $\beta I I$ abundance. Sci Signal 7: ra16, 2014.

26. Iwata-Kajihara T, Sumimoto H, Kawamura N, et al: Enhanced cancer immunotherapy using STAT3-depleted dendritic cells with high Th1-inducing ability and resistance to cancer cell-derived inhibitory factors. J Immunol 187: 27-36, 2011.

27. Chen Y, Wang J, Wang X, et al: STAT3, a poor survival predicator, is associated with lymph node metastasis from breast cancer. J Breast Cancer 16: 40-49, 2013

28. Bednarek I, Sypniewski D, Gawlik N, Galilejczyk A and Goraus K: The efficiency of silencing expression of the gene coding STAT3 transcriptional factor and susceptibility of bladder cancer cells to apoptosis. Contemp Oncol (Pozn) 16: 316-321, 2012.

29. Couto JP, Daly L, Almeida A, et al: STAT3 negatively regulates thyroid tumorigenesis. Proc Natl Acad Sci USA 109: E2361-E2370, 2012.

30. Dong L, Li Y, Cao J, et al: FGF2 regulates melanocytes viability through the STAT3-transactivated PAX3 transcription. Cell Death Differ 19: 616-622, 2012.

31. Coleman SJ, Chioni AM, Ghallab M, et al: Nuclear translocation of FGFR1 and FGF2 in pancreatic stellate cells facilitates pancreatic cancer cell invasion. EMBO Mol Med 6: 467-481, 2014.

32. Lee HJ, Seo AN, Park SY, et al: Low prognostic implication of fibroblast growth factor family activation in triple-negative breast cancer subsets. Ann Surg Oncol 21: 1561-1568, 2014.

33. Meng QH, Xu E, Hildebrandt MA, et al: Genetic variants in the fibroblast growth factor pathway as potential markers of ovarian cancer risk, therapeutic response, and clinical outcome. Clin Chem 60: 222-232, 2014

34. Huang C, Huang R, Chang W, et al: The expression and clinical significance of pSTAT3, VEGF and VEGF-C in pancreatic adenocarcinoma. Neoplasma 59: 52-61, 2012.

35. Yu XM, Lo CY, Chan WF, Lam KY, Leung P and Luk JM: Increased expression of vascular endothelial growth factor $\mathrm{C}$ in papillary thyroid carcinoma correlates with cervical lymph node metastases. Clin Cancer Res 11: 8063-8069, 2005.

36. Karaca Z, Tanriverdi F, Unluhizarci K, et al: VEGFR1 expression is related to lymph node metastasis and serum VEGF may be a marker of progression in the follow-up of patients with differentiated thyroid carcinoma. Eur J Endocrinol 164: 277-284, 2011.

37. Hai-yun S, Deng-ting C and Shou-yi D: Preoperative and postoperative serum VEGF-C levels and its clinical significance. Xi Bao Yu Fen Zi Mian Yi Xue Za Zhi 26: 903-904, 2010 (In Chinese).

38. Emoto N, Onose H, Sugihara H, Minami S, Shimizu K and Wakabayashi I: Fibroblast growth factor-2 free from extracellular matrix is increased in papillary thyroid carcinomas and Graves' thyroids. Thyroid 8: 491-497, 1998. 